\title{
Production of recombinant proteins through sequestration in chloroplasts: a strategy based on nuclear transformation and post-translational protein import
}

\author{
Thangarasu Muthamilselvan ${ }^{1}$. Jung Sun $\mathrm{Kim}^{2}$ - Gangwon Cheong ${ }^{3} \cdot$ Inhwan Hwang $^{1}$
}

Received: 25 March 2019 / Revised: 6 May 2019 / Accepted: 21 May 2019 / Published online: 28 May 2019

(c) Springer-Verlag GmbH Germany, part of Springer Nature 2019

\begin{abstract}
Recently, plants have emerged as a lucrative alternative system for the production of recombinant proteins, as recombinant proteins produced in plants are safer and cheaper than those produced in bacteria and animal cell-based production systems. To obtain high yields in plants, recombinant proteins are produced in chloroplasts using different strategies. The first strategy is based on chloroplast transformation, followed by gene expression and translation in chloroplasts. This has proven to be a powerful approach for the production of proteins at high levels. The second approach is based on nuclear transformation, followed by post-translational import of proteins from the cytosol into chloroplasts. In the nuclear transformation approach, foreign genes are stably integrated into the nuclear genome or transiently expressed in the nucleus by non-integrating T-DNA. Although this approach also has great potential for protein production at high levels, it has not been thoroughly investigated. In this review, we focus on nuclear transformation-based protein expression and its subsequent sequestration in chloroplasts, and summarize the different strategies used for high-level production of recombinant proteins. We also discuss future directions for further improvements in protein production in chloroplasts through nuclear transformation-based gene expression.
\end{abstract}

Keywords Chloroplast $\cdot$ Plant-produced recombinant proteins $\cdot$ Nuclear transformation of foreign genes $\cdot$ Agrobacteriummediated transformation $\cdot$ Protein import

\section{Introduction}

Advances in molecular biology and genetic engineering, together with the development of various biotechnological tools, have enabled the production of a large number of protein drugs, vaccines, and industrial enzymes, such as $\alpha$-amylase and phytase, in living organisms (Bandaranayake and Almo 2014; Chen 2012; Islam et al. 2018; Obembe

Communicated by Neal Stewart.

Inhwan Hwang

ihhwang@postech.ac.kr

1 Division of Integrative Biosciences and Biotechnology, Pohang University of Science and Technology, Pohang, South Korea

2 Department of Agricultural Biotechnology, National Institute of Agricultural Sciences, Rural Development Administration, Jeonju 54874, South Korea

3 Department of Life Science, Gyeongsang National University, Jinju, South Korea et al. 2011; Sohn et al. 2018; Tang and Zhao 2009; Tschofen et al. 2016; Xu et al. 2011). Bacteria and animal cells have been widely used as platforms for the production of various proteins, which are currently available in the market as commercialized products (Bandaranayake and Almo 2014; Chen 2012). However, these conventional production systems have certain shortcomings; for example, the animal cellbased system is expensive and cannot be operated on a large scale (Egelkrout et al. 2012). Recently, plants have received increasing attention as a new platform for the production of recombinant proteins. Compared with animal cells and bacteria, plants have various advantages as protein production systems, such as easy scalability, and potentially zero risk of contamination with human pathogens and bacterial toxins. These advantages have led to the concept of green pharmaceuticals, i.e., pharmaceuticals produced in plants (Egelkrout et al. 2012; Obembe et al. 2011; Xu et al. 2011; Zoschke and Bock 2018).

In plant cells, recombinant proteins are often expressed in subcellular organelles (Jang et al. 1999). In leaf tissues, one of the most attractive organelles for protein production is the 
chloroplast. In mesophyll cells of mature leaves, the number of chloroplasts is as high as 100, and the area occupied by a chloroplast is second to that occupied by a vacuole (Daniell et al. 2016; Zoschke and Bock 2018). Moreover, unlike vacuoles, chloroplasts have neutral $\mathrm{pH}$ and minimally active proteolysis (Zoschke and Bock 2018). Thus, chloroplasts are ideal for the storage of recombinant proteins in plant cells. This is supported by the Rubisco protein complex, which is stored in chloroplasts and accounts for half of the total cellular proteins in leaf tissues (Vitlin Gruber and Feiz 2018).

Two different approaches have been developed for recombinant protein production in chloroplasts. One approach is based on the integration of the target gene into the chloroplast genome via homologous recombination, followed by transcription and translation in chloroplasts (Adem et al. 2017). Many excellent review papers have been published on this topic (Adem et al. 2017; Chebolu and Daniell 2009; Daniell et al. 2009, 2016; Jin and Daniell 2015; Olejniczak et al. 2016; Scotti et al. 2012); therefore, this approach is not covered in this review but referred to in these papers.

The second approach is based on nuclear transformation; the transgene is either transiently expressed in the plant cell nucleus or stably integrated into the plant nuclear genome, and the translated protein is imported into the chloroplasts from the cytosol (Cui et al. 2011; Gelvin 2003; Zahin et al. 2016). Like the chloroplast transformation method, this approach also has great potential for the production of foreign proteins in chloroplasts; however, it has not received much attention and has not been fully explored for the production of recombinant proteins. Compared with research conducted on the chloroplast transformation approach, only a few research articles have been published on import of recombinant protein into the chloroplast using nuclear transformation technologies. In this review, we summarize advantages and weaknesses of the nuclear transformationbased approach for the import of recombinant proteins from the cytosol to the chloroplast for the large-scale production.

\section{Expression of target genes in the nucleus for protein production in chloroplasts}

Agrobacterium tumefaciens, a phytopathogenic Gram-negative soil bacterium, has been used for delivering transgenes into the nucleus of plant cells. A substantial region of the tumor-inducing (Ti) plasmid of Agrobacterium, referred to as the transfer DNA (T-DNA), is integrated into the host plant genome (Gelvin 2003; Hwang et al. 2017a; Smith and Townsend 1907). Plant transformation is performed using the binary vector system, which was developed based on the T-DNA of Agrobacterium. Bacterial genes within the T-DNA are replaced with the gene of interest and delivered into the host nucleus during transformation. Recently, it has been shown that Agrobacterium can be used to transform not only tobacco but also a large number of other plant species (Guo et al. 2018). Thus, there is almost no restriction on plant species for foreign gene expression via nuclear transformation. To produce recombinant proteins in chloroplasts via nuclear transformation, two different approaches have been developed, based on the status of the transgene in the nucleus. In the first approach, the transgene is stably integrated into the nuclear genome, whereas, in the second approach, the transgene is transiently expressed in the nucleus by the unintegrated T-DNA (Fig. 1). Although both approaches rely on nuclear gene expression, there are several differences that distinguish these two approaches when used for recombinant protein production in plants.

\section{Stable expression system for foreign proteins}

Stable integration of the transgene in the nuclear genome requires the generation of transgenic plants. First, the transgene is introduced into a binary vector. Subsequently, the binary vector carrying the transgene is introduced into A. tumefaciens, which transfers the T-DNA from the binary vector into the host cells, where the T-DNA is integrated into the nuclear genome. In this approach, a marker gene is integrated into the nuclear genome along with the gene of interest, thereby allowing the screening of transformed cells from which transgenic plants are regenerated. This is followed by the selection of homozygous plants for the production of proteins encoded by transgenes. The advantage of this approach is that the production of plant biomass expressing the recombinant proteins is as simple as growing plants in a greenhouse, and does not require any additional steps such as agroinfiltration. Elite lines selected on the basis of performance are propagated via seeds, thus requiring minimal maintenance. Thus, this approach is the most suitable for large-scale protein production. However, the disadvantage of this approach is that the generation of homozygous lines is a time-consuming process. In addition, this approach can be used for the production of transgenic in only a limited number of plant species. Furthermore, individual transgenic plants produced using this approach show high variability in transgene expression. To obtain transgenic plants expressing the transgene at high levels, it is necessary to screen a large number of independent transgenic plants; therefore, this method is labor intensive. Moreover, the expression level of a single copy of the transgene stably integrated into the host genome is generally lower than that resulting from the transient transgene expression post-agroinfiltration, and the expression of transgenes is often silenced after several generations. To address these problems, many new approaches have been developed recently. These include the use of matrix attachment sequences (to ensure transgene expression independent of the integration site; (Halweg et al. 
Fig. 1 Stable or transient expression of foreign genes from the nuclear genome and post-translational import of foreign proteins into chloroplasts. Two methods are used for transgene expression following nuclear transformation. In the first method, T-DNA harboring the transgene is stably integrated into the nuclear genome, thereby giving rise to transgenic plants, and in the second method, the non-integrated transgene is expressed from the nucleus and subsequently proteins are synthesized on cytosolic ribosomes and posttranslationally imported into chloroplasts

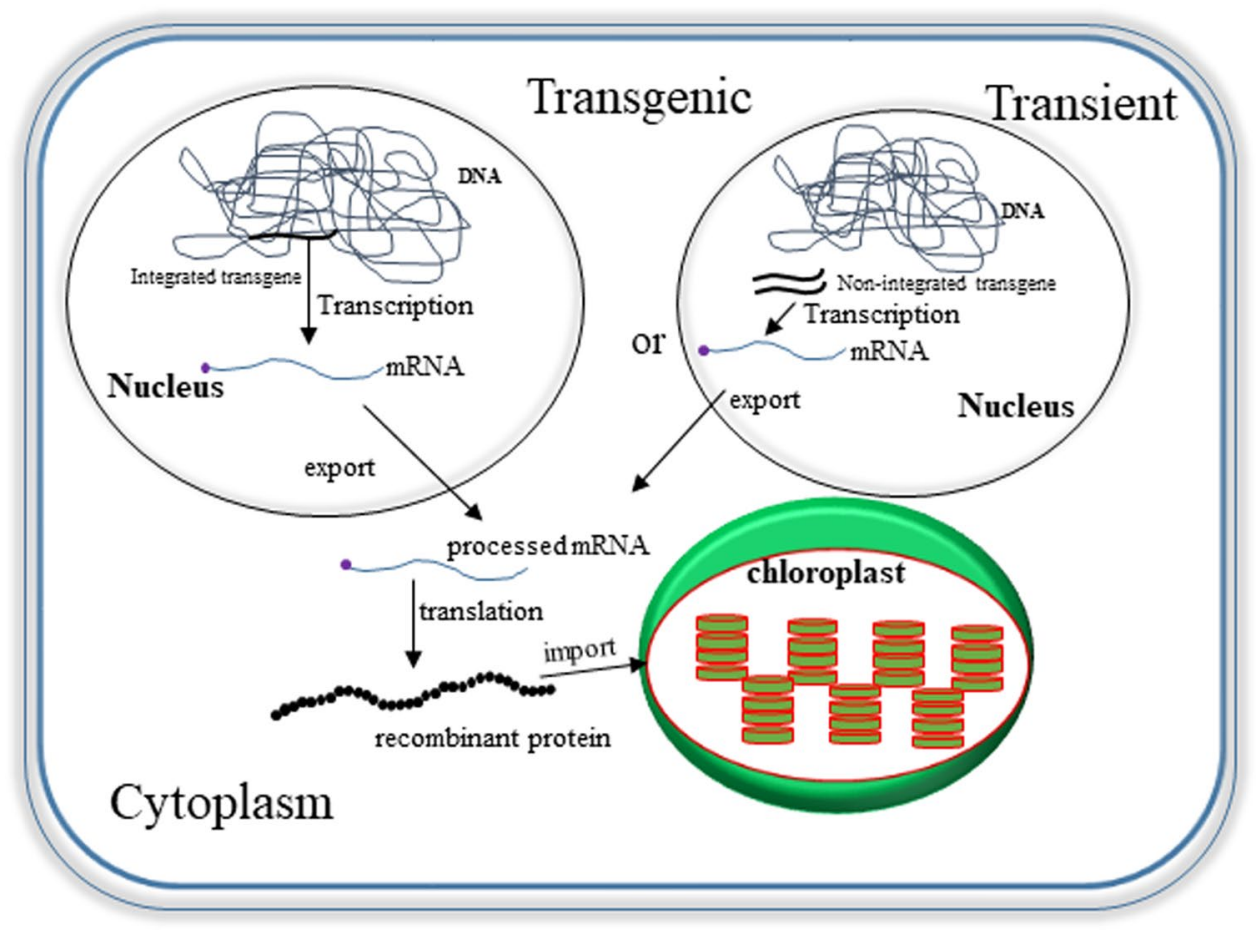

2005), strong artificial transcription factors (Li et al. 2017; Lowder et al. 2018), inducible gene silencing suppressors, transcription terminators [with high transcription efficiency; (Csorba et al. 2015)], and 5' untranslated regions [with high translational efficiency; (Kim et al. 2013; Li et al. 2017)]. These advances in the expression of transgenes in the host cell nucleus provide a new strategy for high-level protein production in chloroplasts. In addition, according to the current regulation of genetically modified (GM) plants, it is important to identify homozygous transgenic plants carrying a single-copy insertion of the target gene in the intergenic region. Thus, this approach would best fit the current regulation of GM plants for commercialization.

\section{Transient expression system for foreign proteins}

In this approach, the transgene carried by the non-integrating T-DNA system is only transiently present and expressed in the nucleus of leaf cells post-agroinfiltration through mutant Agrobacterium strains (Fig. 1; Gelvin 2003; Kapila et al. 1997; Sheludko 2008). Thus, this approach is not subject to the limitations of transgene integration in the nuclear genome, such as the lengthy process of transgenic plant selection and the effect of the insertion site on transgene expression (Marillonnet et al. 2005; Sheludko et al. 2007; Sheludko 2008; Vaquero et al. 1999). Using this approach, recombinant proteins can be produced within a few days, typically ranging from 3 to 10 day post-agroinfiltration. Thus, the transient expression system is a rapid method for the production of recombinant proteins compared with the stable transformation system. This approach would be particularly suitable for the production of recombinant vaccines in the event of viral disease outbreaks (Chen et al. 2013). The most important advantage of this approach is the high-level protein production compared with the transgenic approach. The transgene copy number in the nucleus is higher in transiently transformed plants than that in singlecopy transgenic plants, leading to higher protein production (Huang and Mason 2004; Komarova et al. 2010; Sheludko 2008). If the binary vector is based on DNA or RNA viruses, the copy number of the target gene or mRNA, respectively, is amplified greatly, thus leading to high-level protein production (Cañizares et al. 2006; Huang et al. 2009; Lico et al. 2008; Marillonnet et al. 2005; Sheludko 2008). For example, the transient expression of a foreign gene using the MagnICON vector, an RNA virus-based expression vector, followed by import of translated foreign proteins into chloroplasts led to the expression level as high as that obtained with the transplastomic expression approach (Marillonnet et al. 2004, 2005). Moreover, gene silencing can be avoided by the coexpression of gene silencing suppressors (Csorba et al. 2015). Furthermore, it is possible to express multiple genes simultaneously, enabling the production of protein complexes composed of hetero-multimeric subunits (Pineo et al. 2013).

However, this approach also has certain disadvantages compared with the transgenic approach. In the transient expression approach, a new Agrobacterium culture is needed each time recombinant protein production is required, and plants must be infiltrated individually. Moreover, 
agroinfiltration is a laborious process compared with the sowing of seeds in the transgenic approach. Thus, the overall cost of biomass production using the transient expression approach is much higher than that using the transgenic approach. Another point of concern is that the efficiency of protein production varies among individual plants transformed in one experiment and among plants transformed in different experiments; this could be a major problem when the consistency of protein production is important, as in the case of pharmaceuticals (Fujiuchi et al. 2016). Another disadvantage of this method is the use of Agrobacterium, a Gram-negative species of bacteria. Recently, plant systems have been advocated as a safe system with no or very low level of endotoxins (Islam et al. 2018). However, with the use of Agrobacterium, the plant system is no longer considered an endotoxin-free system, and additional processing is needed to remove endotoxins of Agrobacterium origin from the purified proteins. It should be noted that the abovementioned issues are not specific to protein production in chloroplasts; instead, these issues represent the general problems of this approach when used for recombinant protein production in plants.

\section{Protein import into chloroplasts from the cytosol after translation}

Successful application of nuclear transformation for foreign protein production in chloroplasts is critically dependent on the efficiency of protein import into chloroplasts after translation in the cytosol. Thus, it is important to understand how a large amount of protein is imported into chloroplasts from the cytosol for high-level protein accumulation. The capacity to deliver proteins from the cytosol to the chloroplast may not be a limiting factor, because more than 3000 different proteins are imported into chloroplasts from the cytosol (Jarvis 2008; Lee et al. 2013; Li and Chiu 2010). Moreover, the three most highly abundant proteins in plant leaf cells, Rubisco large subunit (RbcL), Rubisco small subunit $(\mathrm{RbcS})$, and chlorophyll a/b-binding protein $(\mathrm{Cab})$, occur in chloroplasts. Two of these three proteins, $\mathrm{RbcS}$ and $\mathrm{Cab}$, are imported into chloroplasts from the cytosol. Thus, the first step for high-level protein production in chloroplasts via nuclear transformation is to identify a signal sequence that is highly efficient in delivering foreign proteins into chloroplasts. Since transit peptides are cargo-independent (Zhang and Glaser 2002; Zybailov et al. 2008), the selection of a highly efficient transit peptide is critical for the accumulation of proteins at high levels in chloroplasts. Transit peptides of $\mathrm{RbcS}$ and $\mathrm{Cab}$ proteins have been widely used for protein production in chloroplasts (Shen et al. 2017) examples below). However, transit peptides are highly variable in sequence and protein import efficiency. Small sequence motifs present in the transit peptide play crucial roles in the efficiency of protein import into chloroplasts. Thus, it is possible that the modulation of these sequence motifs affects the protein import efficiency (Lee et al. 2006, 2018). Consistent with this possibility, a recent report showed that modified chloroplast transit peptides improve the import of diverse E. coli enzymes such as EcTSR and EcGCL into rice chloroplasts (Shen et al. 2017). Thus, the identification of transit peptides with high protein import efficiency could potentially enhance recombinant protein production in chloroplasts via nuclear transformation.

Another important issue in transit peptide-mediated delivery of proteins into chloroplasts is the removal of transit peptide from the target recombinant protein after import into chloroplasts. During or after import of precursors into chloroplasts, the $\mathrm{N}$-terminal transit peptide is cleaved off by signal propeptide peptidases (SPPs) within chloroplasts (Chen and Li 2007; Richter and Lamppa 1998). However, the entire signal sequence is not removed from the target proteins after import, because the cleavage site of SPPs is located within the transit peptide (Emanuelsson et al. 1999; Lee et al. 2008). Thus, target proteins contain many extra residues derived from the transit peptide. It is possible that the N-terminal region relative to the SPP cleavage site of the transit peptide can be used as a signal sequence for delivering proteins into chloroplasts. However, this may decrease the import efficiency, as the sequence downstream of the cleavage site also contributes to the import efficiency (Lee et al. 2008). Another approach for completely removing the transit peptide from the target protein after import into chloroplasts is to include a new proteolytic cleavage site between the transit peptide and target protein. For example, enterokinase removes the remaining region of the transit peptide when the enterokinase recognition site is inserted between the transit peptide and $\mathrm{N}$-terminus of the target protein. Enterokinase cleaves the C-terminal end of the cleavage site, leaving no extra residues (Hosfield and Lu 1999; Skala et al. 2013).

\section{Demonstration of foreign gene expression and sequestration of translated proteins into chloroplasts via nuclear transformation technologies}

\section{Stable expression of foreign proteins}

Several studies report successful production of recombinant proteins in chloroplasts using the nuclear transformation strategy (Table 1). These include production of $\beta$-glucuronidase (GUS), green fluorescent protein (GFP), and $E$. coli phosphoenolpyruvate synthetase and xylanase in various plant species such as petunia, potato, rice, and tobacco (Hyunjong et al. 2005; Kavanagh et al. 1988; Köhler 
Table 1 List of proteins expressed via nuclear transformation, followed by transit peptide-mediated protein import into chloroplasts

\begin{tabular}{|c|c|c|c|c|}
\hline Transit peptide & Protein & Expression level & Host plant & References \\
\hline $\mathrm{Cab}$ & $\beta$-glucuronidase & & Tobacco & (Kavanagh et al. 1988) \\
\hline $\mathrm{RbcS}$ & $\begin{array}{l}\text { Glutathione reductase, phosphinothricin } \\
\text { acetyl transferase }\end{array}$ & & Tobacco & (Creissen et al. 1995) \\
\hline recA & GFP & & Tobacco & (Köhler et al. 1997) \\
\hline $\mathrm{RbcS}$ & Phosphoenolpyruvate synthetase & $0.1 \% \mathrm{TSP}$ & Potato & (Panstruga et al. 1997) \\
\hline $\mathrm{RbcS}$ & GFP & $10 \% \mathrm{TSP}$ & Rice & (Jang et al. 1999) \\
\hline gbss & GFP strictosidine synthase & & Tobacco cell lines & (Hoppmann et al. 2002) \\
\hline $\mathrm{RbcS}$ & Polyhydroxybutyrate & & Maize & (Zhong et al. 2003) \\
\hline $\mathrm{RbcS}$ & Xylanase & $4.8 \% \mathrm{TSP}$ & Arabidopsis & (Hyunjong et al. 2005) \\
\hline $\mathrm{RbcS}$ & Cry1Ac & $2 \% \mathrm{TSP}$ & Rice & (Kim et al. 2009) \\
\hline $\mathrm{Cab}$ & Endoglucanase Cel5A & $5.2 \% \mathrm{TSP}$ & Tobacco & (Kim et al. 2010) \\
\hline rCTP & EcTSR EcGCL & & Rice & (Shen et al. 2017) \\
\hline $\mathrm{RbcS}$ & HPV16-L1 & $11 \% \mathrm{TSP}$ & Tobacco & (Maclean et al. 2007) \\
\hline $\mathrm{RbcS}$ & p24 & $230 \mu \mathrm{g} / \mathrm{kg}$ & Tobacco & (Meyers et al. 2008) \\
\hline
\end{tabular}

$R b c S$ Rubisco small subunit, $C a b$ chlorophyll $a / b$-binding protein, gbss granule-bound starch synthase, $r e c A$ chloroplast transit peptide, $r C T P$ rice chloroplast transit peptide, HPV16 human papilloma virus type 16, GFP green fluorescent protein, TSP total soluble protein

et al. 1997; Panstruga et al. 1997). The purified GUS protein from petunia showed 95\% bioactivity (Kavanagh et al. 1988). Transit peptides of Arabidopsis or rice RbcS or Cab proteins were used for the delivery of these proteins to chloroplasts. The level of protein accumulation in chloroplasts showed high variability, depending on the protein and plant species. For example, in potato, E. coli phosphoenolpyruvate synthetase accounted for approximately $0.1 \%$ of the total soluble protein (TSP) (Panstruga et al. 1997). However, xylanase and GFP were expressed at much higher levels, reaching approximately $4.5 \%$ and $10 \%$ of the TSP in transgenic tobacco and rice plants, respectively. In addition, HPV16-L1 or p24 antigen produced in transgenic tobacco plants accounted for $11 \%$ of the TSP or $230 \mu \mathrm{g} / \mathrm{kg}$ (fresh weight; fw) of plant biomass (Maclean et al. 2007; Meyers et al. 2008). To date, the level of HPV16-L1 protein produced in transgenic tobacco plants is the highest level of protein production achieved in chloroplasts through nuclear transformation (Jang et al. 1999). Hoppmann et al. (2002) used tobacco cell lines to produce GFP and Catharanthus roseus strictosidine synthase (Str1); these proteins accumulated to very high levels when delivered to chloroplasts using the transit peptide of potato granule-bound starch synthase (gbss). In addition, several enzymes have been produced using this approach. Polyhydroxybutyrate (PHB) enzymes of Alcaligenes eutrophus were produced in chloroplasts of transgenic maize (Zhong et al. 2003). Hyperthermostable endoglucanase Cel5A and a protein encoded by the truncated $c r y 1 A c$ gene were produced to approximately $5.2 \%$ and $2 \%$ of the TSP in transgenic tobacco and rice plants, respectively (Kim et al. 2009, 2010). Moreover, Cel5A produced in chloroplasts was highly efficient in the degradation of its substrate, carboxy-methyl cellulose (CMC) (Kim et al. 2010). In these three studies, the transit peptide of RbcS was used to deliver proteins to chloroplasts. Together, these studies support the potential of recombinant protein production in chloroplasts using stable transformation.

\section{Transient expression of foreign proteins}

Recently, transient expression of foreign genes, followed by protein import into chloroplasts, was used for high-level protein production in plants (Table 2). For example, bioactive human growth hormone (hGH) and Norwalk virus capsid proteins were expressed to $0.1 \%$ of the TSP and approximately $0.1 \mathrm{mg} / \mathrm{kg}$ (fw) of plant biomass, respectively (Gils et al. 2005). The truncated Gag (p17/p24) and p24 capsid

Table 2 List of proteins expressed in tobacco via agroinfiltration, followed by protein import into chloroplasts using the transit peptide of the small subunit of Rubisco $(\mathrm{RbcS})$

\begin{tabular}{lll}
\hline Protein & Expression level & References \\
\hline GFP & & (Marillonnet et al. 2004) \\
Human growth hormone & $0.1 \% \mathrm{TSP}$ & (Gils et al. 2005) \\
HPV16-L1 & $4.5 \% \mathrm{TSP}$ & (Maclean et al. 2007) \\
NVCP & $0.11 \mathrm{mg} / \mathrm{g}$ & (Santi et al. 2008) \\
p24 & $1 \mathrm{mg} / \mathrm{kg}$ & (Meyers et al. 2008) \\
HPV16-L1 & $1710 \mathrm{mg} / \mathrm{kg}$ & (Pineo et al. 2013) \\
HPV16-L1 & $250 \mathrm{mg} / \mathrm{kg}$ & (Zahin et al. 2016) \\
LALF32-51-E7 & $0.44 \% \mathrm{TSP}$ & (Yanez et al. 2017) \\
LALF32-51-E7 & & (Yanez et al. 2018) \\
\hline
\end{tabular}

HPV16 human papilloma virus type $16, N V C P$ Norwalk virus capsid protein, GFP green fluorescent protein, TSP total soluble protein 
subunit proteins of human immunodeficiency virus type 1 (HIV-1) were produced at very high levels, exceeding $1 \mathrm{mg} /$ $\mathrm{kg}$ (fw) of plant biomass (Meyers et al. 2008). Moreover, p17/p24 proteins boosted T-cell and humoral responses in mice that had been primed with a gag DNA vaccine (Meyers et al. 2008). Another example of a viral protein produced in chloroplasts is HPV16 L1; Maclean et al. (2007) produced $137 \mathrm{mg}$ HPV16 L1 per kg (fw) of plant biomass. Moreover, L1 proteins were properly assembled into virus-like particles (VLPs) in N. benthamiana, which are highly immunogenic in target animals and can be developed into effective vaccines; thus, this result demonstrates the potential of this approach for vaccine development. Another study showed further improvement in the productivity of HPV16 L1 VLPs using the MagnICON vector (Zahin et al. 2016); using this vector, $250 \mathrm{mg}$ HPV16 L1 protein was produced per $\mathrm{kg}$ ( $\mathrm{fw}$ ) of plant biomass. VLPs of the human papilloma virus (HPV) are composed of both $\mathrm{L} 1$ and $\mathrm{L} 2$ proteins, and are thought to exhibit higher immunogenicity than L1 alone (Zahin et al. 2016). Indeed, coexpression of HPVI6 L1 and L2 genes encoding the major and minor coat proteins, respectively, resulted in the accumulation of $1710 \mathrm{mg}$ HPV 16 VLPs per $\mathrm{kg}$ (fw) of plant biomass (Pineo et al. 2013). In other studies, Yanez et al. $(2017,2018)$ demonstrated the production of HPV16 E7 to high levels in plants. Together, these studies demonstrate that transient transgene expression, followed by protein import into chloroplasts, is a powerful approach for high-level recombinant protein production in plants.

\section{Potential production of $\mathbf{N}$-glycosylated proteins in chloroplasts}

Chloroplasts originally evolved from cyanobacteria and, therefore, do not have the ability to synthesize glycosylated proteins. However, many pharmaceutical proteins are $\mathrm{N}$-glycosylated (Gomord et al. 2010; Solá and Griebenow 2010). These $\mathrm{N}$-glycans are important for the stability of proteins. Moreover, in certain cases, $\mathrm{N}$-glycans are crucial for the biological activity of proteins. Proteins are $\mathrm{N}$-glycosylated in the endoplasmic reticulum (ER) via a system involving a large number of proteins (Ceriotti et al. 1998). However, it has been shown that certain chloroplast proteins such as $\alpha$-type carbonic anhydrases and amylases are $\mathrm{N}$-glycosylated (Burén et al. 2011; Chen et al. 2004; Faye and Daniell 2006; Lehtimäki et al. 2015). Moreover, $\alpha$-carbonic anhydrases in Arabidopsis are one of the most abundant proteins in chloroplasts, suggesting that a large amount of foreign proteins can be transported to chloroplasts via this route. Recent studies show that $\mathrm{N}$-glycosylated chloroplast proteins use a special route to reach chloroplasts (Lehtimäki et al. 2015). These proteins are first targeted to the ER and then to the Golgi apparatus. In the ER, proteins are $N$-glycosylated, and
$\mathrm{N}$-glycans are modified to the complex type in the Golgi apparatus (Chen et al. 2004; Villarejo et al. 2005). Subsequently, $N$-glycosylated proteins are diverted from the Golgi apparatus to chloroplasts. The exact mechanism of trafficking from the Golgi to the chloroplast has not been elucidated. These data suggest that this pathway can be used for the import of $\mathrm{N}$-glycosylated foreign proteins in chloroplasts in a large-scale production. To use this pathway for recombinant protein production, proteins must be produced via nuclear transformation. However, this pathway has not yet been used for the import of foreign proteins in chloroplasts.

\section{Conclusion and perspectives}

Proteins are one of the most useful biomaterials. Advances in molecular biology and genetic engineering have enabled the use of proteins in a wide range of applications such as biopharmaceuticals, vaccines, antibodies, and industrial enzymes. With the sequencing of the entire genomes of numerous organisms, ranging from bacteria to human, the source of protein-coding genes has increased substantially. In addition, recombinant DNA technology has enabled the generation of new genes by de novo synthesis or by combining domains from naturally existing genes. To ensure that recombinant proteins are used in a wide range of applications, the method of protein production must be economical and highly scalable. For these reasons, plants have recently gained popularity as a platform for recombinant protein production. Chloroplasts are considered excellent subcellular organelles in plants for the production of recombinant proteins at high levels. Two main approaches have been developed for protein production in chloroplasts: chloroplast transformation, which has emerged as a highly cost-effective method, and nuclear transformation, followed by protein import into chloroplasts, which has not been explored extensively for the production of foreign proteins. Transient transgene expression via agroinfiltration has been shown to produce target proteins at extremely high levels in chloroplasts, suggesting that nuclear transformation-based expression is also an excellent option for protein production by import of translated recombinant proteins into chloroplasts. In contrast to transient expression, stable integration of a foreign gene in the nuclear genome has a clear disadvantage for protein production, because proteins are expressed from a single copy of the gene. However, it is still possible to achieve a high level of protein production from limited copy numbers of genes. For example, in Arabidopsis, only two copies of the RbcS gene have been used for the accumulation of $\mathrm{RbcS}$ protein to extremely high levels in chloroplasts. New innovative approaches are needed for improving the level of protein production in chloroplasts. One possible 
approach is to use $\mathrm{RbcS}$ as a fusion partner to derive the production of foreign proteins at high levels. Accumulation of foreign proteins has been improved by several fold using the full-length RbcS protein as a fusion partner rather than using only the transit peptide of RbcS (Hwang et al. 2017b). Based on the design principle of transit peptides, synthetic transit peptides with high efficiency in protein import into chloroplasts could be generated to deliver a large amount of protein into chloroplasts (Lee and Hwang 2018). In addition, the nuclear transformation approach could be explored for producing $N$-glycosylated proteins in chloroplasts. Although considerable research has been conducted for demonstrating the production of recombinant proteins at high levels in chloroplasts by importing them into chloroplasts, no recombinant proteins produced in chloroplasts have yet been commercialized. We expect that this promising approach will soon be used for the production of commercial protein products.

Author contribution statement TMS, JSK, GC and IHH conceived and co-wrote this review. TMS drew Fig. 1.

Acknowledgements This work was supported by the Woojangchoon Project (PJ0109532018) of Rural Development Agency, Korea. Thangarasu Muthamil Selvan was supported by Korea Research Fellowship Program through the National Research Foundation of Korea (NRF) funded by the Ministry of Science and ICT (Grant Number 2016H1D3A1938045).

\section{Compliance with ethical standards}

Conflict of interest The authors declare that they have no conflict of interest.

\section{References}

Adem M, Beyene D, Feyissa T (2017) Recent achievements obtained by chloroplast transformation. Plant Methods 13:30

Bandaranayake AD, Almo SC (2014) Recent advances in mammalian protein production. FEBS Lett 588:253-260

Burén S, Ortega-Villasante C, Blanco-Rivero A, Martínez-Bernardini A, Shutova T, Shevela D, Messinger J, Bako L, Villarejo A, Samuelsson G (2011) Importance of post-translational modifications for functionality of a chloroplast-localized carbonic anhydrase (CAH1) in Arabidopsis thaliana. PLoS One 6:e21021

Cañizares MC, Liu L, Perrin Y, Tsakiris E, Lomonossoff GP (2006) A bipartite system for the constitutive and inducible expression of high levels of foreign proteins in plants. Plant Biotechnol J 4:183-193

Ceriotti A, Duranti M, Bollini R (1998) Effects of $N$-glycosylation on the folding and structure of plant proteins. J Exp Bot 49:1091-1103

Chebolu S, Daniell H (2009) Chloroplast-derived vaccine antigens and biopharmaceuticals: expression, folding, assembly and functionality. Curr Top Microbiol Immunol 332:33-54
Chen R (2012) Bacterial expression systems for recombinant protein production: E. coli and beyond. Biotechnol Adv 30:1102-1107

Chen KY, Li H (2007) Precursor binding to an 880-kDa Toc complex as an early step during active import of protein into chloroplasts. Plant J 49:149-158

Chen M-H, Huang L-F, H-m Li, Chen Y-R, Yu S-M (2004) Signal peptide-dependent targeting of a rice $\alpha$-amylase and cargo proteins to plastids and extracellular compartments of plant cells. Plant Physiol 135:1367-1377

Chen Q, Lai H, Hurtado J, Stahnke J, Leuzinger K, Dent M (2013) Agroinfiltration as an effective and scalable strategy of gene delivery for production of pharmaceutical proteins. Adv Tech Biol Med $1: 103$

Creissen G, Reynolds H, Xue Y, Mullineaux P (1995) Simultaneous targeting of pea glutathione reductase and of a bacterial fusion protein to chloroplasts and mitochondria in transgenic tobacco. Plant J 8:167-175

Csorba T, Kontra L, Burgyán J (2015) Viral silencing suppressors: tools forged to fine-tune host-pathogen coexistence. Virology 479:85-103

Cui C, Song F, Tan Y, Zhou X, Zhao W, Ma F, Liu Y, Hussain J, Wang Y, Yang G, He G (2011) Stable chloroplast transformation of immature scutella and inflorescences in wheat (Triticum aestivum L.). Acta Biochim Biophys Sin 43:284-291

Daniell H, Singh ND, Mason H, Streatfield SJ (2009) Plant-made vaccine antigens and biopharmaceuticals. Trends Plant Sci 14:669-679

Daniell H, Lin CS, Yu M, Chang WJ (2016) Chloroplast genomes: diversity, evolution, and applications in genetic engineering. Genome Biol 17:134

Egelkrout E, Rajan V, Howard JA (2012) Overproduction of recombinant proteins in plants. Plant Sci 184:83-101

Emanuelsson O, Nielsen H, Von Heijne G (1999) ChloroP, a neural network-based method for predicting chloroplast transit peptides and their cleavage sites. Protein Sci 8:978-984

Faye L, Daniell H (2006) Novel pathways for glycoprotein import into chloroplasts. Plant Biotechnol J 4:275-279

Fujiuchi N, Matoba N, Matsuda R (2016) Environment control to improve recombinant protein yields in plants based on Agrobacterium-mediated transient gene expression. Front Bioeng Biotechnol 4:23

Gelvin SB (2003) Agrobacterium-mediated plant transformation: the biology behind the "gene-jockeying" tool. Microbiol Mol Biol Rev 67:16-37

Gils M, Kandzia R, Marillonnet S, Klimyuk V, Gleba Y (2005) High-yield production of authentic human growth hormone using a plant virus-based expression system. Plant Biotechnol J 3:613-620

Gomord V, Fitchette AC, Menu-Bouaouiche L, Saint-Jore-Dupas C, Plasson C, Michaud D, Faye L (2010) Plant-specific glycosylation patterns in the context of therapeutic protein production. Plant Biotech J 8:564-587

Guo M, Ye J, Gao D, Xu N, Yang J (2018) Agrobacterium-mediated horizontal gene transfer: mechanism, biotechnological application, potential risk and forestalling strategy. Biotechnol Adv 37(1):259-270

Halweg C, Thompson WF, Spiker S (2005) The Rb7 matrix attachment region increases the likelihood and magnitude of transgene expression in tobacco cells: a flow cytometric study. Plant Cell 17:418-429

Hoppmann V, Di Fiore S, Zimmermann S, Emans N (2002) The potato granule bound starch synthase chloroplast transit peptide directs recombinant proteins to plastids. J Plant Physiol 159:1061

Hosfield T, Lu Q (1999) Influence of the amino acid residue downstream of (Asp) 4Lys on enterokinase cleavage of a fusion protein. Anal Biochem 269:10-16 
Huang Z, Mason HS (2004) Conformational analysis of hepatitis B surface antigen fusions in an Agrobacterium-mediated transient expression system. Plant Biotech J 2:241-249

Huang Z, Chen Q, Hjelm B, Arntzen C, Mason H (2009) A DNA replicon system for rapid high-level production of virus-like particles in plants. Biotechnol Bioeng 103:706-714

Hwang H-H, Yu M, Lai E-M (2017a) Agrobacterium-mediated plant transformation: biology and applications. Arabidopsis Book 15:e0186

Hwang I, Kang HJ, Sohn EJ, Lee YJ, Berggren P-O, Yoo YJ, Kim JY (2017b) Method of highly expressing target protein from plants using $\mathrm{RbcS}$ fusion protein and method of preparing composition for oral adminstration of medical protein using target protein expression plant body. In: US patent application publication No. US2017/0335361 A1

Hyunjong B, Lee D-S, Hwang I (2005) Dual targeting of xylanase to chloroplasts and peroxisomes as a means to increase protein accumulation in plant cells. J Exp Bot 57:161-169

Islam MR, Kwak JW, Js Lee, Hong SW, Khan MRI, Lee Y, Lee Y, Lee SW, Hwang I (2018) Cost-effective production of tag-less recombinant protein in Nicotiana benthamiana. Plant Biotechnol J 17(6):1094-1105

Jang I-C, Nahm BH, Kim J-K (1999) Subcellular targeting of green fluorescent protein to plastids in transgenic rice plants provides a high-level expression system. Mol Breed 5:453-461

Jarvis P (2008) Targeting of nucleus-encoded proteins to chloroplasts in plants. New Phytol 179:257-285

Jin S, Daniell H (2015) The engineered chloroplast genome just got smarter. Trends Plant Sci 20:622-640

Kapila J, De Rycke R, Van Montagu M, Angenon G (1997) An Agrobacterium-mediated transient gene expression system for intact leaves. Plant Sci 122:101-108

Kavanagh TA, Jefferson RA, Bevan MW (1988) Targeting a foreign protein to chloroplasts using fusions to the transit peptide of a chlorophyll $a / b$ protein. Mol Gen Genet 215:38-45

Kim EH, Suh SC, Park BS, Shin KS, Kweon SJ, Han EJ, Park SH, Kim YS, Kim JK (2009) Chloroplast-targeted expression of synthetic cry1Ac in transgenic rice as an alternative strategy for increased pest protection. Planta 230:397-405

Kim S, Lee D-S, Choi IS, Ahn S-J, Kim Y-H, Bae H-J (2010) Arabidopsis thaliana Rubisco small subunit transit peptide increases the accumulation of thermotoga maritima endoglucanase Cel5A in chloroplasts of transgenic tobacco plants. Transgenic Res 19:489-497

Kim Y, Lee G, Jeon E, Ej Sohn, Lee Y, Kang H, Dw Lee, Kim DH, Hwang I (2013) The immediate upstream region of the 5'-UTR from the AUG start codon has a pronounced effect on the translational efficiency in Arabidopsis thaliana. Nucleic Acids Res 42:485-498

Köhler RH, Cao J, Zipfel WR, Webb WW, Hanson MR (1997) Exchange of protein molecules through connections between higher plant plastids. Science 276:2039-2042

Komarova TV, Baschieri S, Donini M, Marusic C, Benvenuto E, Dorokhov YL (2010) Transient expression systems for plantderived biopharmaceuticals. Expert Rev Vaccines 9:859-876

Lee DW, Hwang I (2018) Evolution and design principles of the diverse chloroplast transit peptides. Mol Cells 41:161

Lee DW, Lee S, G-j Lee, Lee KH, Kim S, Cheong G-W, Hwang I (2006) Functional characterization of sequence motifs in the transit peptide of Arabidopsis small subunit of rubisco. Plant Physiol 140:466-483

Lee DW, Kim JK, Lee S, Choi S, Kim S, Hwang I (2008) Arabidopsis nuclear-encoded plastid transit peptides contain multiple sequence subgroups with distinctive chloroplast-targeting sequence motifs. Plant Cell 20:1603-1622
Lee DW, Jung C, Hwang I (2013) Cytosolic events involved in chloroplast protein targeting. Biochim Biophys Acta 1833:245-252

Lee DW, Yoo Y-J, Razzak MA, Hwang I (2018) Prolines in transit peptides are crucial for efficient preprotein translocation into chloroplasts. Plant Physiol 176:663-677

Lehtimäki N, Koskela MM, Mulo P (2015) Posttranslational modifications of chloroplast proteins: an emerging field. Plant Physiol 168:768-775

Li H, Chiu CC (2010) Protein transport into chloroplasts. Annu Rev Plant Biol 61:157-180

Li Z, Zhang D, Xiong X, Yan B, Xie W, Sheen J, Li J-F (2017) A potent Cas9-derived gene activator for plant and mammalian cells. Nat Plants 3:930

Lico C, Chen Q, Santi L (2008) Viral vectors for production of recombinant proteins in plants. J Cell Physiol 216:366-377

Lowder LG, Zhou J, Zhang Y, Malzahn A, Zhong Z, Hsieh T-F, Voytas DF, Zhang Y, Qi Y (2018) Robust transcriptional activation in plants using multiplexed CRISPR-Act2. 0 and mTALE-act systems. Mol Plant 11:245-256

Maclean J, Koekemoer M, Olivier A, Stewart D, Hitzeroth I, Rademacher T, Fischer R, Williamson A-L, Rybicki E (2007) Optimization of human papillomavirus type 16 (HPV-16) L1 expression in plants: comparison of the suitability of different HPV-16 L1 gene variants and different cell-compartment localization. J Gen Virol 88:1460-1469

Marillonnet S, Giritch A, Gils M, Kandzia R, Klimyuk V, Gleba Y (2004) In planta engineering of viral RNA replicons: efficient assembly by recombination of DNA modules delivered by Agrobacterium. Proc Natl Acad Sci USA 101:6852-6857

Marillonnet S, Thoeringer C, Kandzia R, Klimyuk V, Gleba Y (2005) Systemic Agrobacterium tumefaciens-mediated transfection of viral replicons for efficient transient expression in plants. Nat Biotechnol 23:718

Meyers A, Chakauya E, Shephard E, Tanzer FL, Maclean J, Lynch A, Williamson A-L, Rybicki EP (2008) Expression of HIV-1 antigens in plants as potential subunit vaccines. BMC Biotechnol 8:53

Obembe OO, Popoola JO, Leelavathi S, Reddy SV (2011) Advances in plant molecular farming. Biotechnol Adv 29:210-222

Olejniczak SA, Łojewska E, Kowalczyk T, Sakowicz T (2016) Chloroplasts: state of research and practical applications of plastome sequencing. Planta 244:517-527

Panstruga R, Hippe-Sanwald S, Lee Y-K, Lataster M, Lipka V, Fischer R, Liao YC, Häusler RE, Kreuzaler F, Hirsch H-J (1997) Expression and chloroplast-targeting of active phosphoenolpyruvate synthetase from Escherichia coli in Solanum tuberosum. Plant Sci 127:191-205

Pineo CB, Hitzeroth II, Rybicki EP (2013) Immunogenic assessment of plant-produced human papillomavirus type $16 \mathrm{~L} 1 / \mathrm{L} 2$ chimaeras. Plant Biotech J 11:964-975

Richter S, Lamppa GK (1998) A chloroplast processing enzyme functions as the general stromal processing peptidase. Proc Natl Acad Sci USA 95:7463-7468

Santi L, Batchelor L, Huang Z, Hjelm B, Kilbourne J, Arntzen CJ, Chen Q, Mason HS (2008) An efficient plant viral expression system generating orally immunogenic Norwalk virus-like particles. Vaccine 26:1846-1854

Scotti N, Rigano MM, Cardi T (2012) Production of foreign proteins using plastid transformation. Biotechnol Adv 30:387-397

Sheludko YV (2008) Agrobacterium-mediated transient expression as an approach to production of recombinant proteins in plants. Recent Pat Biotechnol 2:198-208

Sheludko Y, Sindarovska Y, Gerasymenko I, Bannikova M, Kuchuk N (2007) Comparison of several Nicotiana species as hosts for highscale Agrobacterium-mediated transient expression. Biotechnol Bioeng 96:608-614 
Shen BR, Zhu CH, Yao Z, Cui LL, Zhang JJ, Yang CW, He ZH, Peng XX (2017) An optimized transit peptide for effective targeting of diverse foreign proteins into chloroplasts in rice. Sci Rep 7:46231

Skala W, Goettig P, Brandstetter H (2013) Do-it-yourself histidinetagged bovine enterokinase: a handy member of the protein engineer's toolbox. J Biotech 168:421-425

Smith EF, Townsend CO (1907) A plant-tumor of bacterial origin. Science 25:671-673

Sohn EJ, Lee Y, Park N, Park M, Kim NH, Park S, Min K, Gu S, Park Y, Song J (2018) Development of plant-produced E2 protein for use as a green vaccine against classical swine fever virus. J Plant Biol 61:241-252

Solá RJ, Griebenow K (2010) Glycosylation of therapeutic proteins. BioDrugs 24:9-21

Tang WL, Zhao H (2009) Industrial biotechnology: tools and applications. Biotechnol J 4:1725-1739

Tschofen M, Knopp D, Hood E, Stöger E (2016) Plant molecular farming: much more than medicines. Annu Rev Anal Chem 9:271-294

Vaquero C, Sack M, Chandler J, Drossard J, Schuster F, Monecke M, Schillberg S, Fischer R (1999) Transient expression of a tumorspecific single-chain fragment and a chimeric antibody in tobacco leaves. Proc Natl Acad Sci USA 96:11128-11133

Villarejo A, Burén S, Larsson S, Déjardin A, Monné M, Rudhe C, Karlsson J, Jansson S, Lerouge P, Rolland N (2005) Evidence for a protein transported through the secretory pathway en route to the higher plant chloroplast. Nat Cell Biol 7:1224

Vitlin Gruber A, Feiz L (2018) Rubisco assembly in the chloroplast. Front Mol Biosci 5:24

Xu J, Ge X, Dolan MC (2011) Towards high-yield production of pharmaceutical proteins with plant cell suspension cultures. Biotechnol Adv 29:278-299

Yanez RJ, Lamprecht R, Granadillo M, Weber B, Torrens I, Rybicki EP, Hitzeroth II (2017) Expression optimization of a cell membrane-penetrating human papillomavirus type 16 therapeutic vaccine candidate in Nicotiana benthamiana. PLoS One 12:e0183177

Yanez RJ, Lamprecht R, Granadillo M, Torrens I, Arcalís E, Stöger E, Rybicki EP, Hitzeroth II (2018) LALF32-51-E7, a HPV-16 therapeutic vaccine candidate, forms protein body-like structures when expressed in Nicotiana benthamiana leaves. Plant Biotechnol J 16:628-637

Zahin M, Joh J, Khanal S, Husk A, Mason H, Warzecha H, S-j Ghim, Miller DM, Matoba N, Jenson AB (2016) Scalable production of HPV16 L1 protein and VLPs from tobacco leaves. PLoS One 11:e0160995

Zhang XP, Glaser E (2002) Interaction of plant mitochondrial and chloroplast signal peptides with the Hsp70 molecular chaperone. Trends Pharmacol Sci 7:14-21

Zhong H, Teymouri F, Chapman B, Maqbool SB, Sabzikar R, ElMaghraby Y, Dale B, Sticklen MB (2003) The pea (Pisum sativum L.) rbcS transit peptide directs the Alcaligenes eutrophus polyhydroxybutyrate enzymes into the maize (Zea mays L.) chloroplasts. Plant Sci 165:455-462

Zoschke R, Bock R (2018) Chloroplast translation: structural and functional organization, operational control, and regulation. Plant Cell 30:745-770

Zybailov B, Rutschow H, Friso G, Rudella A, Emanuelsson O, Sun Q, van Wijk KJ (2008) Sorting signals, N-terminal modifications and abundance of the chloroplast proteome. PLoS One 3:e1994

Publisher's Note Springer Nature remains neutral with regard to jurisdictional claims in published maps and institutional affiliations. 